\title{
A Note on Emptiness for Alternating Finite Automata with a One-Letter Alphabet *
}

\author{
Petr Jančar Zdeněk Sawa \\ Center of Applied Cybernetics, Dept. of Computer Science, \\ Technical University of Ostrava (FEI V̌̌B-TUO) \\ 17. listopadu 15, CZ-70833 Ostrava, Czech Republic \\ \{Petr.Jancar,Zdenek.Sawa\}@vsb.cz
}

\begin{abstract}
We present a new proof of PSPACE-hardness of the emptiness problem for alternating finite automata with a singleton alphabet. This result was shown by Holzer (1995) who used a proof relying on a series of reductions from several papers. The new proof is simple, direct and self-contained.
\end{abstract}

Key words: computational complexity, alternating finite automaton, emptiness

\section{Introduction}

Checking emptiness, i.e. checking whether the language accepted by a given automaton is (non-)empty, is a natural problem studied in automata theory. It is well known that the emptiness problem is PSPACE-complete for alternating finite automata (AFA), the hardness being implied by the PSPACEcompleteness of the universality problem for nondeterministic finite automata. It is probably less well known that the problem 1L-AFA-EMPTINESS, the emptiness problem for AFA with a singleton alphabet, is also PSPACE-hard; this was shown by Holzer in [3], who thus completed the results of [5].

During the conference presentation of [8], Markus Lohrey noted that Holzer's result can help strengthen some presented complexity lower bounds. In fact, it also helps strengthen some results in [4], and Jiří Srba was inspired to use the result in [9].

If one is interested in the actual proof of PSPACE-hardness of

‡ The authors acknowledge the support by the Czech Ministry of Education, Grant No. $1 \mathrm{M} 0567$. 
1L-AFA-Emptiness, it is a bit unpleasant to find that Holzer uses the emptiness problem for so called EP0L systems [7] which was shown to be PSPACEcomplete in [6], where the proof of PSPACE-hardness (solving a long-term open question) uses a series of reductions among several problems, one of these reductions being handled by a reference to [2].

In this note we observe that the PSPACE-hardness of 1L-AFA-EMPTINESS can be shown directly by a "master reduction," and we note that the idea was implicitly present already in the seminal paper on alternation [1]. In fact, a little adjustment of the construction could also serve to show the PSPACE-hardness of all problems in the above mentioned series in [6].

\section{The main observation}

Let us consider a fixed deterministic Turing machine $M$ with space bounded by $f(n)$. For any input $w$ for $M$ we will show how to construct a one-letteralphabet AFA (1L-AFA) $A_{w}$ with $O(f(|w|))$ states so that $M$ accepts $w$ iff $L\left(A_{w}\right) \neq \emptyset$; by $|w|$ we denote the length of $w$.

We start by recalling the basic definitions.

For a set $X$ we use $\operatorname{Bool}^{+}(X)$ to denote the set of (positive) boolean formulas that only use $\wedge$ and $\vee$ as boolean connectives and elements of $X$ as variables. By $[\phi]_{\nu}$ we denote the truth value (0 or 1$)$ of formula $\phi \in \operatorname{Bool}^{+}(X)$ under the boolean assignment $\nu: X \rightarrow\{0,1\}$.

An alternating finite automaton (AFA) is a structure $A=\left(Q, \Sigma, \delta, q_{0}, F\right)$ where $Q$ is the finite set of states, $\Sigma$ is the finite alphabet, $\delta: Q \times \Sigma \rightarrow \mathrm{Bool}^{+}(Q)$ is the transition function, $q_{0}$ is the initial state, and $F \subseteq Q$ is the set of accepting states.

We define the predicate $A c c \subseteq Q \times \Sigma^{*}$ by induction on the length of the second component; $A c c(q, w)$ is to be read as " $A$ starting in $q$ accepts $w . "$

- $\operatorname{Acc}(q, \varepsilon)$ iff $q \in F$.

- $\operatorname{Acc}(q, a w)$ iff $[\delta(q, a)]_{\nu}=1$ for the boolean assignment $\nu$ satisfying $\left(\nu\left(q^{\prime}\right)=1 \Leftrightarrow \operatorname{Acc}\left(q^{\prime}, w\right)\right)$ for all $q^{\prime} \in Q$.

AFA $A$ accepts the language $L(A)=\left\{w \in \Sigma^{*} \mid A c c\left(q_{0}, w\right)\right\}$.

When $|\Sigma|=1$, we say that $A$ is a $1 \mathrm{~L}-\mathrm{AFA}$ (1L being read "one letter").

We are interested in the problem 1L-AFA-Emptiness:

INSTANCE: 1L-AFA $A$.

Question: Is $L(A)=\emptyset$ ?

A deterministic Turing machine (deciding a problem, or accepting a language) 
is a structure $M=\left(Q, \Sigma, \Gamma, \delta, q_{0}, q_{a c c}, q_{r e j}\right)$ where $Q$ is the finite set of (control) states, $\Sigma$ is the finite input alphabet, $\Gamma$ is the finite tape alphabet where $\Sigma \subseteq \Gamma$, $\delta:\left(Q-\left\{q_{a c c}, q_{\text {rej }}\right\}\right) \times \Gamma \rightarrow Q \times \Gamma \times\{-1,0,+1\}$ is the transition function, and $q_{0}, q_{a c c}, q_{r e j} \in Q$ are the initial state, the accepting final state and the rejecting final state, respectively. The tape alphabet $\Gamma$ contains a special blank symbol $\square \notin \Sigma$. We assume that $M$ starts with scanning the tape cell with the leftmost symbol of an input word $w \in \Sigma^{+}$and never moves left from that cell. W.l.o.g. we only consider nonempty input words.

Technically we view the tape cells as numbered by nonnegative integers, i.e. by elements of $\mathbb{N}=\{0,1,2, \ldots\}$. A configuration $C$ is then a function $C: \mathbb{N} \rightarrow \Delta$ where $\Delta=\Gamma \cup(Q \times \Gamma)$; the state and the head position are determined by the pair $C(j) \in(Q \times \Gamma)$. Given a (nonempty) input $w=a_{1} a_{2} \ldots a_{n}$, the initial configuration $C_{0}^{w}$ is defined as $C_{0}^{w}(1)=\left(q_{0}, a_{1}\right), C_{0}^{w}(j)=a_{j}$ for $2 \leq j \leq n$, and $C_{0}^{w}(j)=\square$ elsewhere.

The computation of $M$ on $w$ is the (finite or infinite) sequence of configurations $C_{0}^{w}, C_{1}^{w}, C_{2}^{w}, \ldots$ determined by the input $w$ and the transition function $\delta$ in the usual manner. We use the cell 0 for technical convenience; necessarily, $C_{i}^{w}(0)=\square$ for all $i$. It is important that $C_{i+1}^{w}(j)$, for $j \geq 1$, is determined by the triple $\left(C_{i}^{w}(j-1), C_{i}^{w}(j), C_{i}^{w}(j+1)\right)$, not depending on the actual $i, j, w$. For any $z \in \Delta$ we can thus define the following easily constructible set:

$$
\begin{aligned}
& \operatorname{Preds}(z)=\left\{\left(z_{1}, z_{2}, z_{3}\right) \in \Delta^{3}:(\forall i, j, w)\left(\left(C_{i}^{w}(j-1), C_{i}^{w}(j), C_{i}^{w}(j+1)\right)=\right.\right. \\
& \left.\left.\left(z_{1}, z_{2}, z_{3}\right) \text { implies } C_{i+1}^{w}(j)=z\right)\right\} .
\end{aligned}
$$

For technical convenience we also assume that if $M$ enters $q_{a c c}$ then the head scans cell 1 which currently contains $\square$. Thus we can define that $M$ accepts $w$ iff there is $i \in \mathbb{N}$ such that $C_{i}^{w}(1)=\left(q_{a c c}, \square\right)$.

Now we come to the crucial construction. We assume a fixed deterministic Turing machine $M=\left(Q, \Sigma, \Gamma, \delta, q_{0}, q_{a c c}, q_{r e j}\right)$ with space bounded by a function $f$; this means that $M$ can only visit the cells numbered $1,2, \ldots, f(n)$ in the computation starting on an input $w$ with $|w|=n$. The function $f: \mathbb{N} \rightarrow \mathbb{N}$ is supposed to satisfy $f(n) \geq n$ for all $n$, which also means that $C_{i}^{w}(j)=\square$ for $j>f(n)$ in the computation of $M$ on $w$ with $|w|=n$.

For any $w=a_{1} a_{2} \ldots a_{n}$ we define the following 1L-AFA $A_{w}=$ $\left(Q^{\prime},\{\diamond\}, \delta^{\prime}, q_{0}^{\prime}, F^{\prime}\right)$ :

- $Q^{\prime}=\{0,1,2, \ldots, f(n)+1\} \times \Delta($ where $\Delta=\Gamma \cup(Q \times \Gamma))$,

- $q_{0}^{\prime}=\left(1,\left(q_{a c c}, \square\right)\right)$,

- $F^{\prime}=\left\{(j, z) \in Q^{\prime} \mid C_{0}^{w}(j)=z\right\}$,

- for $j \in\{0, f(n)+1\}$ we put $\delta^{\prime}((j, \square), \diamond)=1$ (constantly true) and $\delta^{\prime}((j, z), \diamond)=0$ (constantly false) for $z \neq \square$,

- for $1 \leq j \leq f(n)$ we define:

$$
\delta^{\prime}((j, z), \diamond)=\bigvee_{\left(z_{1}, z_{2}, z_{3}\right) \in \operatorname{Preds}(z)}\left(j-1, z_{1}\right) \wedge\left(j, z_{2}\right) \wedge\left(j+1, z_{3}\right)
$$


The next proposition can be easily shown by induction on $i$. It relates the computation $C_{0}^{w}, C_{1}^{w}, C_{2}^{w}, \ldots$ of the deterministic Turing machine $M$ on $w$ and the predicate $A c c$ corresponding to the AFA $A_{w}=\left(Q^{\prime},\{\diamond\}, \delta^{\prime}, q_{0}^{\prime}, F^{\prime}\right)$.

Proposition 1 For all $i \in \mathbb{N}$ and $(j, z) \in Q^{\prime}$ we have:

$C_{i}^{w}(j)=z \Leftrightarrow \operatorname{Acc}\left((j, z), \diamond^{i}\right)$.

Corollary $2 M$ accepts $w$ iff $\exists i: C_{i}^{w}(1)=\left(q_{a c c}, \square\right)$ iff $\exists i: A c c\left(q_{0}^{\prime}, \diamond^{i}\right)$ iff $L\left(A_{w}\right) \neq \emptyset$.

Theorem 3 1L-AfA-EmPtiness is PSPACE-complete.

PROOF. Any problem $P$ in PSPACE is decided by a deterministic Turing machine $M$ with space bounded by a polynomial $p(n)$. Given such $M$, our (algorithmic) construction of $A_{w}$ can be obviously done in polynomial time, and logarithmic space, wrt $|w|$. Hence every problem in PSPACE is logspacereducible to 1L-AFA-EMPTINESS.

The membership of the emptiness problem in PSPACE is straightforward, even in the case of general AFA; it was shown in [5].

For deriving other PSPACE-hardness results, it is useful to have special simple forms of 1L-AFA for which the emptiness problem is still PSPACE-hard. We present one such form.

We call a $1 L$-AFA $A=\left(Q,\{\diamond\}, \delta, q_{0}, F\right)$ simple if each formula $\delta(q, \diamond)$ is either a variable $q^{\prime}$ or is in the form $q_{1} \wedge q_{2}$ or in the form $q_{1} \vee q_{2}$.

Proposition 4 The emptiness problem for simple $1 L$-AFA is PSPACE-hard.

PROOF. We reduce 1L-AFA-Emptiness to the emptiness problem for simple 1L-AFA.

Let us consider a $1 \mathrm{~L}-\mathrm{AFA} A=\left(Q,\{\diamond\}, \delta, q_{0}, F\right)$. By $f_{q}$ we denote a "fullyparenthesized form" of the formula $\delta(q, \diamond)$; any subformula $f$ of $f_{q}$ is either a variable $q^{\prime}$ or is in the form $\left(f_{1} \wedge f_{2}\right)$ or in the form $\left(f_{1} \vee f_{2}\right)$. By $\operatorname{depth}(f)$ we denote the depth of nesting in $f: \operatorname{depth}(q)=1$ and $\operatorname{depth}\left(f_{1} \wedge f_{2}\right)=$ $\operatorname{depth}\left(f_{1} \vee f_{2}\right)=1+\max \left\{\operatorname{depth}\left(f_{1}\right), \operatorname{depth}\left(f_{2}\right)\right\}$.

Let $m=\max \left\{\operatorname{depth}\left(f_{q}\right): q \in Q\right\}$.

The above 1L-AFA $A$ can be transformed to a simple 1L-AFA $A^{\prime}=$ $\left(Q^{\prime},\{\diamond\}, \delta^{\prime}, q_{0}^{\prime}, F^{\prime}\right)$ defined as follows:

$Q^{\prime}=\left\{\left(1, q_{0}\right)\right\} \cup\left\{(i, f): f\right.$ is a subformula of some $f_{q}$ and $\left.m \geq i \geq \operatorname{depth}(f)\right\}$,

$q_{0}^{\prime}=\left(1, q_{0}\right)$,

$F^{\prime}=\{(1, q): q \in F\}$,

$\delta^{\prime}((1, q), \diamond)=\left(m, f_{q}\right)$,

if $i>\operatorname{depth}(f)$ then $\delta^{\prime}((i, f), \diamond)=(i-1, f)$, 
if $i=\operatorname{depth}(f)$ and $f=\left(f_{1}\right.$ op $\left.f_{2}\right)$ then $\delta^{\prime}((i, f), \diamond)=\left(i-1, f_{1}\right)$ op $\left(i-1, f_{2}\right)$ for op $\in\{\wedge, \vee\}$.

It is obvious that the length of every word in $L\left(A^{\prime}\right)$ is divisible by $m$, and that $\diamond^{j} \in L(A)$ iff $\diamond^{j m} \in L\left(A^{\prime}\right)$. Thus $L(A)=\emptyset$ iff $L\left(A^{\prime}\right)=\emptyset$.

\section{Additional remarks}

We note that the idea of the above construction showing PSPACE-hardness of 1L-AFA-EMPTINESS is implicitly present in the seminal paper [1]. The proof of Theorem 3.4. in [1] shows that, given a deterministic Turing machine $M$ with time (and thus also space) bounded by $f(n)$, we can construct an equivalent alternating Turing machine $M^{\prime}$ with space $O(\log f(n))$. The work of $M^{\prime}$ can be interpreted in our terms as follows: given $w, M^{\prime}$ checks if there is $i \leq f(|w|)$ such that $A_{w}$ (defined wrt $M$ ) accepts $\diamond^{i}$. $M^{\prime}$ cannot construct $A_{w}$ explicitly; it just generates the binary description of a guessed $i \leq f(n)$ and then simulates $i$ steps of $A_{w}$. $M^{\prime}$ has to be able to remember the current state $(j, z)$ of $A_{w}$ but this is no problem since it can use the tape for storing (the binary description of $j$. The ability of $M^{\prime}$ to simulate $A_{w}$ is obvious since the corresponding instructions of $M^{\prime}$ depend only on $M$, not on $w$.

It is also worth to note that 1L-AFA-EMPTINESS can be easily reduced to the emptiness problem for EP0L (as was also observed in [3]), for which the question of PSPACE-hardness had been an open problem until the solution in [6]. The other problems which were shown PSPACE-hard in [6], the emptiness (and other problems) for binary systolic tree automata (BSTA) and for the auxiliary model of "set systems," could be directly derived by using a simple adjustment of the idea used in the construction of $A_{w}$; we now sketch this adjustment.

In the computation $C_{0}^{w}, C_{1}^{w}, C_{2}^{w}, \ldots$ of a deterministic Turing machine $M$ on $w$, the values $C_{i}^{w}(j-1), C_{i}^{w}(j), C_{i}^{w}(j+1)$ can be seen as a substantiation of $C_{i+1}^{w}(j)$; we can think of substantiation rules of the form

$$
(j, z) \Leftarrow\left(\left(j-1, z_{1}\right),\left(j, z_{2}\right),\left(j+1, z_{3}\right)\right)
$$

where $\left(z_{1}, z_{2}, z_{3}\right) \in \operatorname{Preds}(z)$. Looking more closely, we note that each $C_{i+1}^{w}(j)$ can be substantiated by just two elements of $C_{i}^{w}$, namely by the pair $\left(C_{i}^{w}(j), C_{i}^{w}\left(j^{\prime}\right)\right)$ where $C_{i}^{w}\left(j^{\prime}\right) \in Q \times \Gamma$; in the case $C_{i}^{w}(j) \in Q \times \Gamma$ we have $j=j^{\prime}$, a substantiation by one element of $C_{i}^{w}$ - but this can still be viewed as a substantiation by the pair $\left(C_{i}^{w}(j), C_{i}^{w}(j)\right)$ when needed for uniformity. Assuming $M$ has space bounded by $f(n)$, for any input $w$ with $|w|=n$ we can obviously construct $O\left((f(n))^{2}\right)$ substantiation rules

$$
(j, z) \Leftarrow\left(\left(j, z_{1}\right),\left(j^{\prime}, z_{2}\right)\right)
$$


(where $j, j^{\prime} \in\{1,2, \ldots, f(n)\}$ ). We also note the following determinism (important for BSTA): for every pair $\left(\left(j, z_{1}\right),\left(j^{\prime}, z_{2}\right)\right)$ there is at most one $(j, z)$ such that $(j, z) \Leftarrow\left(\left(j, z_{1}\right),\left(j^{\prime}, z_{2}\right)\right)$ is a rule.

\section{References}

[1] A. K. Chandra, D. C. Kozen, L. J. Stockmeyer, Alternation, J. ACM 28 (1) (1981) 114-133.

[2] K. Culik, J. Gruska, A. Salomaa, On a family of L languages resulting from systolic tree automata, Theoretical Comput. Sci. 23 (3) (1983) 231-242.

[3] M. Holzer, On emptiness and counting for alternating finite automata, in: J. Dassow, G. Rozenberg, A. Salomaa (eds.), Proceedings of Developments in Language Theory II (Magdeburg, Germany, 17-21 July 1995), World Scientific, 1996.

[4] P. Jančar, A. Kučera, F. Moller, Z. Sawa, DP lower bounds for equivalencechecking and model-checking of one-counter automata, Information and Computation 188 (2004) 1-19.

[5] T. Jiang, B. Ravikumar, A note on the space complexity of some decision problems for finite automata, Inf. Process. Lett. 40 (1) (1991) 25-31.

[6] A. Monti, A. Roncato, Completeness results concerning systolic tree automata and E0L languages, Inf. Process. Lett. 53 (1) (1995) 11-16.

[7] G. Rozenberg, A. Salomaa, The Mathematical Theory of L Systems, vol. 90 of Pure and Applied Mathematics, Academic Press, 1980.

[8] O. Serre, Parity games played on transition graphs of one-counter processes, in: L. Aceto, A. Ingólfsdóttir (eds.), Proceedings of FOSSACS 2006, vol. 3921 of Lecture Notes in Computer Science, Springer, 2006.

[9] J. Srba, Visibly pushdown automata: From language equivalence to simulation and bisimulation, in: Z. Ésik (ed.), Proceedings of CSL 2006, vol. 4207 of Lecture Notes in Computer Science, Springer-Verlag, 2006. 